increased risk of cesarean section for prolonged labour and cesarean section for fetal distress.

Conclusions Poor maternal vitamin D status was linked to approximately a twofold increased risk of as well as to specific indications for cesarean delivery.

\section{P2-470 REFERENCE VALUES FOR CHILDHOOD BODY MASS INDEX IN THE ERA OF THE OVERWEIGHT EPIDEMIC}

\section{doi:10.1136/jech.2011.142976l.98}

${ }^{1} Y$ Schönbeck, ${ }^{*}{ }^{1} \mathrm{P} \vee$ Dommelen, ${ }^{1,2} \mathrm{~S}$ Van Buuren. ${ }^{1}$ Netherlands Organization for Applied Scientific Research, Leiden, The Netherlands; ${ }^{2}$ University Utrecht, Utrecht, The Netherlands

Introduction Due to the overweight and obesity epidemic, growth references based on recent data may no longer be appropriate for monitoring childhood body mass index (BMI) as they may underestimate the problem. Our aim is to determine the difference in signalling overweight and obesity between the use of up to date references and cut-offs for BMI according to the International Obesity Task Force (IOTF).

Methods The growth references for BMI were constructed using cross-sectional growth data from the Fifth Dutch Growth Study in 2009 ( $n=10129)$. We plotted the IOTF cut-offs against the Dutch growth references for ages $2-18 \mathrm{y}$. Moreover, we compared the prevalence of overweight and obesity according to IOTF with respectively the +1.6 and $+2.7 \mathrm{SD}$ score (SDS) on the Dutch growth references. These SDS cut-offs correspond to a BMI of 25 and $30 \mathrm{~kg} /$ $\mathrm{m}^{2}$ at age $18 \mathrm{y}$ in the Third Dutch Growth Study in 1980, which was part of the sample to construct the IOTF cut-offs.

Results The IOTF cut-offs for overweight and obesity were lower than +1.6 and +2.7 SDS at all ages. The difference between IOTF and the cut-offs for SDS ranged from -1.8 to -2.5 SDS for overweight and -1.2 to -2.3 SDS for obesity.

Conclusion Using up to date population based references for BMI with recommended SDS cut-offs for Dutch children results in an underestimation of the problem. In countries with substantial overweight and obesity, we recommend using pre-epidemic references or cut-offs, such as proposed by the International Obesity Task Force.

\section{P2-471 LIVER FUNCTION RELATED VIRUSES AND DIABETES IN CHINA}

doi:10.1136/jech.2011.142976l.99

${ }^{1,2} \mathrm{C}$ M Schooling, ${ }^{*} \mathrm{C} O$ Jiang, ${ }^{3} \mathrm{~W}$ S Zhang, ${ }^{2} \mathrm{~T}$ H Lam, ${ }^{4} \mathrm{~K}$ K Cheng, ${ }^{2} \mathrm{G} \mathrm{M}$ Leung. ${ }^{1}$ CUNY, New York, USA; ${ }^{2}$ The University of Hong Kong, Hong Kong, China; ${ }^{3}$ Guangzhou Number 12 Hospital, Guangzhou, China; ${ }^{4}$ The University of Birmingham, Birmingham, UK

Introduction Compared to western populations, diabetes mellitus is prevalent in China despite a relatively non-obese population. Exposures beyond lifestyle and genetics may be relevant. We hypothesised that liver infections, common in China, increase vulnerability to diabetes, via the physiological consequences of poor liver function.
Methods We used multivariable regression to examine the adjusted associations of alanine transaminase (ALT) and aspartate transaminase (AST) with diabetes in the Guangzhou Biobank Cohort Study phase 1 (2003-2004) for 10121 older ( $\geq 50$ years) Chinese and in NHANES III for 16854 people. We similarly examined the associations of liver function related viruses (number of hepatitis $A$ $\mathrm{B}, \mathrm{C}$ and $\mathrm{E}$ antibodies or of hepatitis $\mathrm{A}$, hepatitis $\mathrm{C}$ and herpes simplex virus 1) with ALT, AST, diabetes and death from diabetes in NHANES III. As a control, we examined associations for viruses unrelated to liver function (cytomegalovirus, herpes simplex virus 2 and human herpes virus 8).

Results ALTwas positively associated with diabetes in both settings, adjusted for age, sex, socio-economic position, smoking, alcohol and adiposity. Similarly adjusted, liver function related virus antibodies were associated with ALT $(0.18$ SDs, $95 \%$ CI 0.10 to 0.26 for $2+$ compared with none) and diabetes (OR $1.43,95 \%$ CI 1.02 to 2.02 ), but viruses unrelated to liver function were not.

Conclusion Prior exposure to endemic viruses damaging liver function could be an additional factor contributing to diabetes in China, which public health efforts infection control efforts may already be addressing. Prevention strategies may need to be contextually specific.

\section{P2-472 FACTORS AFFECTING NEWBORN CARE PRACTICES IN BANGLADESH}

doi:10.1136/jech.2011.142976l.100

M Shahjahan,* K Fatema, S J Mumu, A Afroz. Bangladesh Institute of Health Sciences, Dhaka, Bangladesh

Introduction Newborn care has important implication in achieving MDG-4 because among infant deaths two-thirds die within 1 month of the birth in Bangladesh. The objective of this paper is to identify the associated factors which affect newborn-care practices in Bangladesh.

Methods This paper used the birth history data of BDHS 2007-which is a nationally representative sample survey. Twostage stratified cluster- sampling design was adopted. A total number of 6150 mothers were interviewed through systematic random sampling.

Results The mean age of mothers was 18.0 (SD \pm 3.2 ) years. Little over $12 \%$ pregnant women received at least one antenatal check-up during the pregnancy. About $85 \%$ of deliveries were conducted at home and $71 \%$ of them were attended by untrained persons. During cord cutting $87 \%$ used clean instrument and $34 \%$ reported to have their first bath immediately after delivery. Twelve percent mother initiated breast feeding within half an hour of the delivery. Logistic regression analysis suggests that maternal education is an important determinant of early breast feeding and new born care. Secondary or higher levels of maternal education were associated with early breast feeding ( $O R=2.9$, CI 1.5 to 5.5). For instance, mothers who had secondary and higher education were almost three times more likely to be aware of the newborn care practices as opposed to mothers who had no education.

Conclusion Since two-thirds of infants die before reaching 1 month, it is recommended that through creating awareness about newborn care practices of rural mothers, infant mortality can be significantly reduced. 